\title{
Continuity of the Shafer-Vovk-Ville Operator
}

\author{
Natan T'Joens, Gert de Cooman, and Jasper De Bock \\ Ghent University, ELIS, SYSTeMS \\ \{natan.tjoens, gert.decooman, jasper.debock\}@ugent.be
}

\begin{abstract}
Kolmogorov's axiomatic framework is the best-known approach to describing probabilities and, due to its use of the Lebesgue integral, leads to remarkably strong continuity properties. However, it relies on the specification of a probability measure on all measurable events. The game-theoretic framework proposed by Shafer and Vovk does without this restriction. They define global upper expectation operators using local betting options. We study the continuity properties of these more general operators. We prove that they are continuous with respect to upward convergence and show that this is not the case for downward convergence. We also prove a version of Fatou's Lemma in this more general context. Finally, we prove their continuity with respect to point-wise limits of two-sided cuts.
\end{abstract}

\section{Introduction}

The most common approach to probability theory is the measure-theoretic framework that originates in Kolmogorov's work [4]. Its popularity is largely due to its interpretational neutrality and the elegant mathematical properties resulting from the use of measure theory. However, this framework requires the definition of a probability measure on all measurable events. Although this is often overlooked, it presents a major drawback, because the actual specification of these probabilities is far from trivial in many practical applications. Hence, the mathematical results are elegant, but the underlying assumptions are very strong.

For dealing with stochastic processes, a more general and intuitive approach was proposed by Shafer and Vovk [5]. Their so-called game-theoretic framework is based on the idea of a supermartingale: a specific way to gamble on the successive outcomes of the process.

In our present contribution, we study the continuity properties of the upper (and therefore also lower) expectation operators that appear in this framework. Our main results are that they are continuous with respect to upward, but not downward, convergence of uniformly bounded below sequences, and continuous with respect to particular limits of two-sided cuts. From our upward convergence result, we also derive a generalised version of Fatou's Lemma.

Due to length constraints, proofs and intermediate results are relegated to the appendix of an extended online version of this paper, available on ArXiv [8] 


\section{Preliminaries}

We denote the set of all natural numbers, without 0 , by $\mathbb{N}$, and let $\mathbb{N}_{0}:=\mathbb{N} \cup\{0\}$. The set of extended real numbers is denoted by $\overline{\mathbb{R}}:=\mathbb{R} \cup\{+\infty,-\infty\}$. The set of positive real numbers is denoted by $\mathbb{R}_{>0}$ and the set of non-negative real numbers by $\mathbb{R}_{\geq 0}$.

We consider sequences of uncertain states $X_{1}, X_{2}, \ldots, X_{n}, \ldots$ where the state $X_{k}$ at each discrete time $k \in \mathbb{N}$ takes a value in some non-empty finite set $\mathscr{X}$, called the state space. We call any $x_{1: n}:=\left(x_{1}, \ldots, x_{n}\right) \in \mathscr{X}_{1: n}:=\mathscr{X}^{n}$, for $n \in \mathbb{N}_{0}$, a situation and we denote the set of all situations by $\mathscr{X}^{*}:=\cup_{n \in \mathbb{N}_{0}} \mathscr{X}_{1: n}$. So any finite string of possible values for a sequence of consecutive states is called a situation. In particular, the unique empty string $x_{1: 0}$, denoted by $\square$, is called the initial situation: $\mathscr{X}_{1: 0}:=\{\square\}$.

An infinite sequence of state values $\omega$ is called a path and the set of all paths is called the sample space $\Omega:=\mathscr{X}^{\mathbb{N}}$. For any path $\omega \in \Omega$, the initial sequence that consists of its first $n$ state values is a situation in $\mathscr{X}_{1: n}$ that is denoted by $\omega^{n}$. The $n$-th state is denoted by $\omega_{n} \in \mathscr{X}$.

\section{Game-Theoretic Upper Expectations}

In order to deal with stochastic processes mathematically, we use variables. A global variable, or simply variable, $f$ is a map on the set $\Omega$ of all paths. An (extended) real variable $f$ associates an (extended) real number $f(\omega)$ with any path $\omega$. The set of all extended real variables is denoted by $\overline{\mathbb{V}}$. For any natural $k \leq \ell$, we use $X_{k: \ell}$ to denote the variable that, for every path $\omega$, returns the tuple $X_{k: \ell}(\omega):=\left(\omega_{k}, \ldots, \omega_{\ell}\right)$. As such, the state $X_{k}=X_{k: k}$ at any discrete time $k$ can also be regarded as a variable.

A collection of paths $A \subseteq \Omega$ is called an event. The indicator $\mathbb{I}_{A}$ of an event $A$ is defined as the variable that assumes the value 1 on $A$ and 0 elsewhere. With any situation $x_{1: n}$, we associate the cylinder event $\Gamma\left(x_{1: n}\right):=\left\{\omega \in \Omega: \omega^{n}=x_{1: n}\right\}$ : the set of all paths $\omega \in \Omega$ that go through the situation $x_{1: n}$. For a given $n \in \mathbb{N}_{0}$, we call a variable $f n$-measurable if it is constant on the cylinder events $\Gamma\left(x_{1: n}\right)$ for all $x_{1: n} \in \mathscr{X}_{1: n}$, that is, if we can write $f=\tilde{f} \circ X_{1: n}=\tilde{f}\left(X_{1: n}\right)$ for some map $\tilde{f}$ on $\mathscr{X}^{n}$. We will then use the notation $f\left(x_{1: n}\right)$ for its constant value $f(\omega)$ on all paths $\omega \in \Gamma\left(x_{1: n}\right)$.

Variables are inherently uncertain objects and, therefore, we need a way to model this uncertainty. We will do this by means of upper and lower expectations, which requires the introduction of gambles.

For any non-empty set $\mathscr{Y}$, we define a gamble $f$ on $\mathscr{Y}$ as a bounded real map on $\mathscr{Y}$. It is then typically interpreted as an uncertain reward $f(y)$ when the outcome of some 'experiment', assuming values in $\mathscr{Y}$, is $y \in \mathscr{Y}$. The set of all gambles on $\mathscr{Y}$ is denoted by $\mathbb{G}(\mathscr{Y})$. In particular, a gamble on $\Omega$ is a bounded real variable. When $\mathscr{Y}=\mathscr{X}$, we call the gamble $f$ a local gamble.

A coherent upper expectation $\overline{\mathrm{E}}$ on the set $\mathbb{G}(\mathscr{Y})$ is defined as a real functional on $\mathbb{G}(\mathscr{Y})$ that satisfies the following coherence axioms $[9,2.6 .1]$ : 
E1. $\overline{\mathrm{E}}(f) \leq \sup f$ for all $f \in \mathbb{G}(\mathscr{Y})$;

E2. $\overline{\mathrm{E}}(f+g) \leq \overline{\mathrm{E}}(f)+\overline{\mathrm{E}}(g)$ for all $f, g \in \mathbb{G}(\mathscr{Y})$;

E3. $\overline{\mathrm{E}}(\lambda f)=\bar{\lambda} \overline{\mathrm{E}}(f)$ for all $f \in \mathbb{G}(\mathscr{Y})$ and real $\lambda \geq 0$.

$\overline{\mathrm{E}}(f)$ can be interpreted as some subject's minimum selling price for the gamble $f \in \mathbb{G}(\mathscr{Y})$ on $\mathscr{Y}$. Alternatively, one can also consider the conjugate lower expectation, defined by $\underline{\mathrm{E}}(f):=-\overline{\mathrm{E}}(-f)$ for all $f \in \mathbb{G}(\mathscr{Y})$. It clearly suffices to focus on only one of the two functionals. We will work with upper expectations.

In an imprecise probability tree we attach to each situation $x_{1: n} \in \mathscr{X}^{*}$ a local probability model characterised by a coherent upper expectation $\overline{\mathrm{Q}}\left(\cdot \mid x_{1: n}\right)$ on the set $\mathbb{G}(\mathscr{X})$ of all local gambles on the next state $X_{n+1}$. These local probability models $\overline{\mathrm{Q}}\left(\cdot \mid x_{1: n}\right)$ are usually known, as, in most practical cases, they can be elicited fairly easily from a subject or learned from data. They express a subject's beliefs or knowledge about the next possible state. However, gathering information or eliciting beliefs about a variable that depends on multiple states or even entire paths is not that straightforward. Therefore, the question arises how we can extend the local probability models (on single states) towards global probability models (on entire paths).

To answer this question, we first need to introduce the concepts of a process and a gamble process. A process $\mathscr{L}$ is a map defined on $\mathscr{X}^{*}$. A real process associates a real number $\mathscr{L}(s) \in \mathbb{R}$ with any situation $s \in \mathscr{X}^{*}$. A real process is called positive (non-negative) if it is positive (non-negative) in every situation. With any real process $\mathscr{L}$ we associate a sequence of $n$-measurable gambles $\left\{\mathscr{L}_{n}\right\}_{n \in \mathbb{N}_{0}}$ : for all $n \in \mathbb{N}_{0}$, we let $\mathscr{L}_{n}(\omega):=\mathscr{L}\left(\omega^{n}\right)$ for all $\omega \in \Omega$ or, equivalently, $\mathscr{L}_{n}:=\mathscr{L} \circ X_{1: n}=\mathscr{L}\left(X_{1: n}\right)$. A gamble process $\mathscr{D}$ is a process that associates with any situation $x_{1: n} \in \mathscr{X}^{*}$ a local gamble $\mathscr{D}\left(x_{1: n}\right) \in \mathbb{G}(\mathscr{X})$. With any real process $\mathscr{L}$, we can associate a gamble process $\Delta \mathscr{L}$, called its process difference. For any situation $x_{1: n}$ the corresponding gamble $\Delta \mathscr{L}\left(x_{1: n}\right) \in \mathbb{G}(\mathscr{X})$ is defined by

$$
\Delta \mathscr{L}\left(x_{1: n}\right)\left(x_{n+1}\right):=\mathscr{L}\left(x_{1: n+1}\right)-\mathscr{L}\left(x_{1: n}\right) \text { for all } x_{n+1} \in \mathscr{X} .
$$

We will also use the extended real variables $\lim \inf \mathscr{L} \in \overline{\mathbb{V}}$ and $\lim \sup \mathscr{L} \in \overline{\mathbb{V}}$, defined by:

$$
\liminf \mathscr{L}(\omega):=\liminf _{n \rightarrow+\infty} \mathscr{L}_{n}(\omega) \text { and } \lim \sup \mathscr{L}(\omega):=\limsup _{n \rightarrow+\infty} \mathscr{L}_{n}(\omega)
$$

for all $\omega \in \Omega$. If $\lim \inf \mathscr{L}=\lim \sup \mathscr{L}$, we denote their common value by $\lim \mathscr{L}$.

For a given imprecise probability tree, a supermartingale $\mathscr{M}$ is a real process for which the process difference $\Delta \mathscr{M}$ has a non-positive local upper expectation everywhere: $\overline{\mathrm{Q}}\left(\Delta \mathscr{M}\left(x_{1: n}\right) \mid x_{1: n}\right) \leq 0$ for all $x_{1: n} \in \mathscr{X}^{*}$. In other words, a supermartingale is a process that, according to the local probability models, is expected to decrease. The concept originates in the following 'game-theoretic' argument. Suppose that a forecaster sets minimum selling prices for every gamble $f$ on the next state $X_{n+1}$, i.e. he defines $\overline{\mathrm{Q}}\left(f \mid x_{1: n}\right)$. Non-positive minimum selling prices imply that he is willing to give away these gambles. Suppose now that you take him up on his commitments. The gambles available to you are then exactly the ones with $\overline{\mathrm{Q}}\left(f \mid x_{1: n}\right) \leq 0$. Choosing such an available gamble in 
every situation $x_{1: n} \in \mathscr{X}^{*}$ essentially defines a supermartingale. In this way, we can interpret a supermartingale as a strategy for gambling against a forecaster.

We define supermartingales here as real processes, whereas Shafer and Vovk define them as extended real processes [6]. For any situation $s \in \mathscr{X}^{*}$, such an extended real process allows the possibility for $\Delta \mathscr{M}(s)$ to be an extended real function on $\mathscr{X}$. However, it is not immediately obvious to us how to give a behavioural meaning to such extended real process differences, and we therefore prefer to define supermartingales as real processes whose differences are gambles.

We denote the set of all supermartingales for a given imprecise probability tree by $\overline{\mathbb{M}}$. The set of all bounded below supermartingales is denoted by $\overline{\mathbb{M}}_{\mathrm{b}}$.

We are now ready to introduce the game-theoretic upper expectation.

Definition 1. The upper expectation $\overline{\mathrm{E}}_{\mathrm{V}}(\cdot \mid \cdot)$ is defined by

$$
\overline{\mathrm{E}}_{\mathrm{V}}(f \mid s):=\inf \left\{\mathscr{M}(s): \mathscr{M} \in \overline{\mathbb{M}}_{\mathrm{b}} \text { and }(\forall \omega \in \Gamma(s)) \liminf \mathscr{M}(\omega) \geq f(\omega)\right\},
$$

for all extended real variables $f \in \overline{\mathbb{V}}$ and all $s \in \mathscr{X}^{*}$.

This definition can be interpreted in the following way: the upper expectation of a variable $f$ when in a situation $s$, is the infimum starting capital in the situation $s$ such that, by using the available gambles from $s$ onwards, we are able to end up with a capital that dominates $f$, no matter the path through $s$ taken by the process. Importantly, these upper expectations for global variables are defined in terms of supermartingales, and therefore, derived directly from the local models. Moreover, due to [5, Prop. 8.8], for every situation $s$, the restriction of $\overline{\mathrm{E}}_{\mathrm{V}}(\cdot \mid s)$ to $\mathbb{G}(\Omega)$ satisfies the coherence axioms E1-E3.

Observe that in defining these global upper expectations, we consider supermartingales that are bounded below, because as is shown in [2, Example 1], for extended real variables, the use of unbounded supermartingales leads to undesirable results, whereas Definition 1 does not.

In the remainder of this contribution, we restrict our attention to upper expectations conditional on the initial situation $\square$ and use the notation $\overline{\mathrm{E}}_{\mathrm{V}}(f):=$ $\overline{\mathrm{E}}_{\mathrm{V}}(f \mid \square)$. This facilitates the reading and makes the paper conceptually easier. That being said, we stress that all our arguments are easily extendible to upper expectations conditional on a general situation $s \in \mathscr{X}^{*}$.

\section{Continuity with Respect to Upward Convergence}

The relevance of continuity properties for (upper) expectation functionals is evident. Not only do they provide the mathematical theory with elegance, they also enhance its practical scope. The continuity of the Lebesgue integral, for instance, is one of the reasons why it is the integral of choice for computing expected values associated with a probability measure. Continuity properties provide constructive ways to calculate expectations that otherwise would be difficult or even impossible to calculate numerically. For example, calculating the upper expectation $\overline{\mathrm{E}}_{\mathrm{V}}(f)$ of an extended real variable $f$ directly is typically 
practically impossible if it depends on an infinite number of states. However, if we can find a sequence of simpler functions $\left\{f_{n}\right\}_{n \in \mathbb{N}_{0}}$ that converges in some way to $f$, such that the upper expectation $\overline{\mathrm{E}}_{\mathrm{V}}$ is continuous with respect to this convergence, then we can easily approximate $\overline{\mathrm{E}}_{\mathrm{V}}(f)$ by $\overline{\mathrm{E}}_{\mathrm{V}}\left(f_{n}\right)$, provided $n$ is large enough. If we can find a sequence for which moreover the individual $\overline{\mathrm{E}}_{\mathrm{V}}\left(f_{n}\right)$ can be calculated directly, we obtain a practical method for calculating $\overline{\mathrm{E}}_{\mathrm{V}}(f)$. Unfortunately, it appears little is known at present about the continuity properties of the functional $\overline{\mathrm{E}}_{\mathrm{V}}$; we aim to remedy this situation here.

It is well-known that every coherent upper expectation $\overline{\mathrm{E}}$ is continuous with respect to uniform convergence [3, p.63]: if a sequence of gambles $\left\{f_{n}\right\}_{n \in \mathbb{N}_{0}}$ converges uniformly to a gamble $f$, meaning that $\lim _{n \rightarrow+\infty} \sup \left\{\left|f-f_{n}\right|\right\}=$ 0 , then $\lim _{n \rightarrow+\infty} \overline{\mathrm{E}}\left(f_{n}\right)=\overline{\mathrm{E}}(f)$. Hence, since the restriction of $\overline{\mathrm{E}}_{\mathrm{V}}$ to $\mathbb{G}(\Omega)$ is a coherent upper expectation, it is continuous with respect to the uniform convergence of gambles on $\Omega$. This type of continuity is however fairly weak, because the condition of uniform convergence is a very strong one. Moreover, continuity with respect to pointwise convergence is not directly implied by mere coherence [3, p.63]. The following example demonstrates that, also for the upper expectation operator $\overline{\mathrm{E}}_{\mathrm{V}}$ we are focussing on here, continuity with respect to pointwise convergence, and downward convergence in particular, may fail.

Example 1. Consider, in each situation $x_{1: n} \in \mathscr{X}^{*}$, a completely vacuous model: $\overline{\mathrm{Q}}\left(h \mid x_{1: n}\right)=\max h$ for all local gambles $h \in \mathbb{G}(\mathscr{X})$ on the next state. Then it can be checked easily that $\overline{\mathrm{E}}_{\mathrm{V}}(f)=\sup f$ for all $f \in \overline{\mathbb{V}}$. Now let $\mathscr{X}:=\{0,1\}$, and consider the decreasing sequence of events $A_{n}$, defined by $A_{n}:=\{\omega \in$ $\Omega: \omega_{i}=1$ for all $\left.1 \leq i \leq n\right\} \backslash\{(1,1,1, \ldots)\}$. Then $\lim _{n \rightarrow+\infty} \mathbb{I}_{A_{n}}=0$ pointwise. However, as $\overline{\mathrm{E}}_{\mathrm{V}}\left(\mathbb{I}_{A_{n}}\right)=1$ for all $n \in \mathbb{N}_{0}$, we have that $\lim _{n \rightarrow+\infty} \overline{\mathrm{E}}_{\mathrm{V}}\left(\mathbb{I}_{A_{n}}\right)=1$, whereas $\overline{\mathrm{E}}_{\mathrm{V}}\left(\lim _{n \rightarrow+\infty} \mathbb{I}_{A_{n}}\right)=\overline{\mathrm{E}}_{\mathrm{V}}(0)=0$, so $\overline{\mathrm{E}}_{\mathrm{V}}$ is not continuous with respect to downward pointwise convergence of gambles.

This leads us to the conclusion that, in general, $\overline{\mathrm{E}}_{\mathrm{V}}$ is not continuous with respect to downward - and therefore also pointwise - convergence. Nevertheless, using a version of Lévy's zero-one law [8], we can show that $\overline{\mathrm{E}}_{\mathrm{V}}$ is continuous with respect to upward convergence of extended real variables that are uniformly bounded below, provided that the upper expectation of the limit variable $f$ is finite.

Theorem 1 (Upward Convergence Theorem). Consider any non-decreasing sequence of extended real variables $\left\{f_{n}\right\}_{n \in \mathbb{N}_{0}}$ that is uniformly bounded belowi.e. there is an $M \in \mathbb{R}$ such that $f_{n} \geq M$ for all $n \in \mathbb{N}_{0}$-and any extended real variable $f \in \overline{\mathbb{V}}$ such that $\lim _{n \rightarrow+\infty} f_{n}=f$ pointwise. If moreover $\overline{\mathrm{E}}_{\mathrm{V}}(f)<+\infty$, then

$$
\overline{\mathrm{E}}_{\mathrm{V}}(f)=\lim _{n \rightarrow+\infty} \overline{\mathrm{E}}_{\mathrm{V}}\left(f_{n}\right) \text {. }
$$

The initial idea behind the proof is due to Shafer and Vovk, who proved continuity with respect to non-decreasing sequences of indicator gambles [1, Theorem 6.6]. We have adapted it here to our working with real supermartingales and moreover generalised it to extended real variables.

The following example illustrates the practical relevance of this theorem. 
Example 2. In queuing theory or failure estimation, we are often interested in the time until some event happens and, in particular, in the lower and upper expectation of this time. As we will illustrate here, Theorem 1 provides a method to approximate such upper expectations. The lower expectations can also be approximated, using Theorem 3 further on; see Example 3.

Consider the simple case where $\mathscr{X}:=\{0,1\}$. Suppose we are interested in the expected time until the first ' 1 ' appears. In other words, we are interested in the variable $f$ that returns the number of initial successive ' 0 's in a path:

$$
f(\omega):=\inf \left\{k \in \mathbb{N}: \omega_{k}=1\right\} \text { for all } \omega \in \Omega,
$$

where for $\omega=(0,0,0, \ldots), f(\omega)=\inf \emptyset:=+\infty$. It is typically infeasible to calculate the upper expectation of this variable directly because it depends on entire paths. We can remedy this by considering instead, for every $n \in \mathbb{N}_{0}$, the gamble $f_{n}$, defined by

$$
f_{n}(\omega):=\min \{f(\omega), n\} \text { for all } \omega \in \Omega .
$$

For every $n \in \mathbb{N}_{0}, f_{n}$ is clearly $n$-measurable: it only depends on the value of the first $n$ states. Furthermore, $\left\{f_{n}\right\}_{n \in \mathbb{N}_{0}}$ is bounded below by zero, non-decreasing and converges pointwise to $f$. Provided that $\overline{\mathrm{E}}_{\mathrm{V}}(f)<+\infty$, Theorem 1 therefore implies that $\overline{\mathrm{E}}_{\mathrm{V}}(f)=\lim _{n \rightarrow+\infty} \overline{\mathrm{E}}_{\mathrm{V}}\left(f_{n}\right)$. This allows us to approximate $\overline{\mathrm{E}}_{\mathrm{V}}(f)$ by $\overline{\mathrm{E}}_{\mathrm{V}}\left(f_{n}\right)$, for $n$ sufficiently large. Since the $n$-measurability of $f_{n}$ will typically make the computation of $\overline{\mathrm{E}}_{\mathrm{V}}\left(f_{n}\right)$ feasible, we obtain a practical method for computing $\overline{\mathrm{E}}_{\mathrm{V}}(f)$.

As a direct consequence of our Upward Convergence Theorem, we also obtain the following inequality.

Theorem 2 (Fatou's Lemma). Consider a sequence of extended real variables $\left\{f_{n}\right\}_{n \in \mathbb{N}_{0}}$ that is uniformly bounded below and let $f:=\liminf _{n \rightarrow+\infty} f_{n}$. If $\overline{\mathrm{E}}_{\mathrm{V}}(f)<+\infty$, then

$$
\overline{\mathrm{E}}_{\mathrm{V}}(f) \leq \liminf _{n \rightarrow+\infty} \overline{\mathrm{E}}_{\mathrm{V}}\left(f_{n}\right) .
$$

This result is similar to Fatou's Lemma in measure theory; hence its name. It provides an upper bound on the upper expectation of an extended real variable $f$, in the form of a limit inferior of the upper expectations of any sequence of extended real variables $\left\{f_{n}\right\}_{n \in \mathbb{N}_{0}}$ that is uniformly bounded below and whose limit inferior $\liminf _{n \rightarrow+\infty} f_{n}$ is equal to $f$. Since this last condition is fairly weak, Theorem 2 has wide applicability. In general, the inequality in the statement cannot be reversed, because we do not generally have continuity with respect to pointwise convergence.

\section{Continuity with Respect to Limits of Cuts}

Historically, the framework of imprecise probabilities as described by Walley [9] has only considered gambles rather than unbounded or even extended real variables. One important reason for this is that they allow us to use less involved 
mathematics. Moreover, when considering unbounded or extended variables in practice, we are typically obliged to work with approximating gambles rather than the original variables. Considering these arguments, restricting the functional $\overline{\mathrm{E}}_{\mathrm{V}}$ to gambles would be very tempting, indeed. However, most practically relevant variables in the context of stochastic processes are in fact unbounded and even extended real-valued; consider for instance hitting or stopping times, as in Examples 2 and 3.

In Theorem 1 we have already shown how to approximate upper expectations for bounded below extended variables. The following theorem allows us to approximate upper expectations for general extended real variables by using sequences of non-increasing lower cuts.

Theorem 3. Consider an extended real variable $f \in \overline{\mathbb{V}}$ and, for every $A \in \mathbb{R}$, the variable $f_{A}$ defined by $f_{A}(\omega):=\max \{f(\omega), A\}$ for all $\omega \in \Omega$. Then

$$
\lim _{A \rightarrow-\infty} \overline{\mathrm{E}}_{\mathrm{V}}\left(f_{A}\right)=\overline{\mathrm{E}}_{\mathrm{V}}(f) .
$$

Combining Theorems 1 and 3, we end up with the following result that allows us to move from upper expectations of gambles to upper expectations of general variables. It also fits within the framework of Troffaes and De Cooman [3, Part $2]$, which provides a general approach to extending coherent lower and upper expectations from gambles to real variables.

Theorem 4 (Continuity with respect to cuts). Consider any extended real variable $f \in \overline{\mathbb{V}}$ and, for any $A, B \in \mathbb{R}$ such that $B \geq A$, the gamble $f_{(A, B)}$, defined by

$$
f_{(A, B)}(\omega):=\left\{\begin{array}{l}
B \text { if } f(\omega)>B ; \\
f(\omega) \text { if } B \geq f(\omega) \geq A ; \quad \text { for all } \omega \in \Omega . \\
A \text { if } f(\omega)<A
\end{array}\right.
$$

If $\overline{\mathrm{E}}_{\mathrm{V}}(f)<+\infty$, then

$$
\lim _{A \rightarrow-\infty} \lim _{B \rightarrow+\infty} \overline{\mathrm{E}}_{\mathrm{V}}\left(f_{(A, B)}\right)=\overline{\mathrm{E}}_{\mathrm{V}}(f) .
$$

Example 3. Consider the same state space $\mathscr{X}$ and the same variables $f$ and $f_{n}$ as in Example 2. We have already shown there how to approximate the upper expectation $\overline{\mathrm{E}}_{\mathrm{V}}(f)$ of $f$ by $\overline{\mathrm{E}}_{\mathrm{V}}\left(f_{n}\right)$. Now, we want to approximate the lower expectation $\underline{\mathrm{E}}_{\mathrm{V}}$ - defined by $\underline{\mathrm{E}}_{\mathrm{V}}(g):=-\overline{\mathrm{E}}_{\mathrm{V}}(-g)$ for all $g \in \overline{\mathbb{V}}$ - of $f$. As $\left\{f_{n}\right\}_{n \in \mathbb{N}}$ is an increasing sequence of upper cuts of $f,\left\{-f_{n}\right\}_{n \in \mathbb{N}}$ is a decreasing sequence of lower cuts of $-f$. Hence, it follows from Theorem 3 that $\lim _{n \rightarrow+\infty} \overline{\mathrm{E}}_{\mathrm{V}}\left(-f_{n}\right)=\overline{\mathrm{E}}_{\mathrm{V}}(-f)$, and therefore, using conjugacy, $\lim _{n \rightarrow+\infty}-\underline{\mathrm{E}}_{\mathrm{V}}\left(f_{n}\right)=-\underline{\mathrm{E}}_{\mathrm{V}}(f)$, or, equivalently, $\lim _{n \rightarrow+\infty} \underline{\mathrm{E}}_{\mathrm{V}}\left(f_{n}\right)=\underline{\mathrm{E}}_{\mathrm{V}}(f)$. Hence, in the same way as was described in Example 2, we now also have a constructive method for approximating the lower expectation of the variable $f$. 


\section{Conclusion}

Among the continuity properties derived in this paper, the continuity with respect to cuts is the more remarkable, as it allows us to limit ourselves, for the larger part, to the study of $\overline{\mathrm{E}}_{\mathrm{V}}$ on gambles rather than the study of $\overline{\mathrm{E}}_{\mathrm{V}}$ on extended real variables. Although the functional $\overline{\mathrm{E}}_{\mathrm{V}}$ is not continuous with respect to general downward convergence, it is thus continuous for a particular way of downward convergence: sequences of non-increasing lower cuts. These results hold provided that the upper expectation $\overline{\mathrm{E}}_{\mathrm{V}}(f)$ of the limit variable $f$ is finite. The case where $\overline{\mathrm{E}}_{\mathrm{V}}(f)=+\infty$ is largely left unexplored.

There is also an interesting connection between the game-theoretic functional $\overline{\mathrm{E}}_{\mathrm{V}}$ and the measure-theoretic Lebesgue integral when all local models are assumed precise. It was already pointed out by Shafer and Vovk [5, Chapter 8] that for indicator gambles $\mathbb{I}_{A}$ of events $A$ in the $\sigma$-algebra created by the cylinder events, $\overline{\mathrm{E}}_{\mathrm{V}}\left(\mathbb{I}_{A}\right)$ is equal to the Lebesgue integral of $\mathbb{I}_{A}$ when the global measure is defined according to the Ionescu-Tulcea Theorem [7, p.249]. Using our results, we aim to generalise the connection between both operators and study the extend to which they are equal. We leave this as future work.

Another topic of further research is the continuity of $\overline{\mathrm{E}}_{\mathrm{V}}$ with respect to the pointwise convergence of $n$-measurable gambles. We suspect that, in order to establish the behaviour of $\overline{\mathrm{E}}_{\mathrm{V}}$ with respect to this particular type of convergence, it will pay to investigate the potentially strong link with the concept of natural extension [9] and, as a consequence, the special status of $\overline{\mathrm{E}}_{\mathrm{V}}$ with respect to other extending functionals.

\section{References}

1. Augustin, T., Coolen, F., de Cooman, G., Troffaes, M.: Introduction to Imprecise Probabilities. John Wiley \& Sons, Chichester (May 2014)

2. De Cooman, G., De Bock, J., Lopatatzidis, S.: Imprecise stochastic processes in discrete time: global models, imprecise Markov chains, and ergodic theorems. International Journal of Approximate Reasoning 76(C), 18 - 46 (2016)

3. De Cooman, G., Troffaes, M.: Lower Previsions. Wiley (2014)

4. Kolmogorov, A.: Grundbegriffe der Wahrscheinlichkeitsrechnung. Ergebnisse der Mathematik und ihrer Grenzgebiete, Springer (1933)

5. Shafer, G., Vovk, V.: Probability and Finance. It's Only a Game!, John Wiley \& Sons (2005)

6. Shafer, G., Vovk, V., Takemura, A.: Lévy's zero-one law in game-theoretic probability (2011), arXiv:0905.0254 [math.PR]

7. Shiryaev, A., Wilson, S.: Probability. Graduate Texts in Mathematics, Springer New York (1995)

8. T'Joens, N., de Cooman, G., De Bock, J.: Continuity of the shafer-vovk-ville operator (2018), arXiv:1804.01980 [math.PR]

9. Walley, P.: Statistical Reasoning with Imprecise Probabilities. Chapman and Hall, London (1991) 